\title{
Protein quality control in lung disease: it's all about cloud networking
}

\author{
Silke Meiners ${ }^{1}$ and Catherine M. Greene ${ }^{2}$
}

Affiliations: ${ }^{1}$ Comprehensive Pneumology Center, University Hospital, Ludwig-Maximilians University, Helmholtz Zentrum München, Member of the German Center for Lung Research (DZL), Munich, Germany. ${ }^{2}$ Dept of Medicine, Royal College of Surgeons in Ireland, Education and Research Centre, Beaumont Hospital, Dublin, Ireland.

Correspondence: Silke Meiners, Comprehensive Pneumology Center, Ludwig-Maximilians-Universität, Asklepios Klinik Gauting und Helmholtz Zentrum München, Max-Lebsche-Platz 31, 81377 München, Germany. E-mail: silke.meinersahelmholtz-muenchen.de

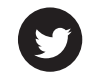

@ERSpublications

Effective protein quality control is central to lung health http://ow.ly/z3zrq

Protein quality control involves the comprehensive management of protein function in the cell and is called "proteostasis" [1]. It ranges from translation and chaperone-assisted three-dimensional folding, interaction with protein partners, signal-induced post-translational modifications to disposal by the proteasome or autophagy pathways.

Dysfunctional protein quality control is emerging as a key pathogenic mechanism for chronic lung diseases. Two major hereditary conformational disorders of the lung, cystic fibrosis and $\alpha_{1}$-antitrypsin $\left(\alpha_{1}\right.$-AT) deficiency, and some familial forms of idiopathic pulmonary fibrosis (IPF) are caused by the expression of mutant and misfolded proteins that disrupt protein homeostasis and drive the onset of pulmonary diseases $[2,3]$. Disturbed proteostasis also causes sporadic respiratory diseases [1, 4]. Cigarette smoke-induced protein misfolding, aberrant proteasomal protein degradation and induction of autophagy have been observed in chronic obstructive pulmonary disease (COPD) patients and smoke-exposed mice $[4,5]$. Dysregulation of autophagy and endoplasmic reticulum (ER) stress have also been implicated in cystic fibrosis, pulmonary arterial hypertension, IPF and other lung diseases [6, 7]. Impairment of protein quality control pathways exacerbates the detrimental effects of environmentally induced protein damage in lung pathogenesis [1].

The European Respiratory Society (ERS) research seminar Protein Quality Control in Lung Disease, held on March 1-2, 2014, at Lake Starnberg in Germany, brought together international experts to develop a comprehensive view of protein quality control in general and in the lung in particular. Understanding the complex interplay of protein misfolding, ER homeostasis and protein degradation as interrelated components of adaptive proteostasis will identify novel therapeutic targets for treatment of pulmonary diseases, as outlined here.

\section{Protein misfolding}

Proteins are synthesised as an amino acid chain that folds into a well-defined three-dimensional structure. A network of molecular chaperones assists protein folding and maintains functional states [8]. Destabilising missense mutations or stress-induced misfolding challenge the proteostasis network and target protein destruction via proteasomal degradation or autophagy. When chaperone-assisted refolding or degradation fails, protein aggregation may occur. Aggregates exert proteotoxicity by interfering with normal proteins and cellular function [8].

Received: June 102014 | Accepted: June 122014

Conflict of interest: None declared.

Copyright @ERS 2014 
In cystic fibrosis, the Phe508del mutation prevents exit of the cystic fibrosis transmembrane conductance regulator (CFTR) from the ER and leads to its premature degradation by the ER quality control systems. Rescuing Phe508del-CFTR has involved corrector compounds such as VX-809 (Vertex, Eysins, Switzerland). Disappointingly, the results of clinical trials are modest $[9,10]$. As pointed out by Margarida Amaral (University of Lisbon, Lisbon, Portugal), synergistic Phe508del-CFTR correction may be achieved by other compounds that target distinct conformational sites, later trafficking stages or cellular checkpoints of the proteostasis network, as recently shown using a druggable genome small interfering RNA library [11]. Unbalancing the proteostasis network with a single mutant protein may also apply to novel rare $\alpha_{1}$-AT variants with altered protein structure, as suggested by Mila Ljujic (Institute of Molecular Genetics and Genetic Engineering, Belgrade, Serbia) [12]. Mark Hipp from the Max Planck Institute of Biochemistry in Munich, Germany, provided evidence for a complete collapse of protein homeostasis: above a certain threshold, misfolded aggregated proteins indirectly inhibited overall protein degradation by the proteasome [13]. Misfolded proteins also sequester chaperones that are then missing for other essential folding and transport functions [14]. William Balch (The Scripps Research Institute, La Jolla, CA, USA) elegantly extended the current protein quality control network concept by proposing dynamic and multidimensional proteostasis network "clouds". A cloud represents a quinary level (Q-state) of structural control to locally and dynamically modify structure and interactions of a protein [15]. At the cellular level, such "Q-clouds" are present in Qbodies that represent local and dynamic cytoplasmic compartments containing chaperone-complexed misfolded proteins as well as degradation components such as the proteasome (fig. 1) [16]. This concept adds a novel spatial and dynamic level to the proteostasis network: upsetting the Q-cloud with single point-mutated proteins may result in a chronic proteotoxic crisis contributing to the pathology of chronic lung diseases.

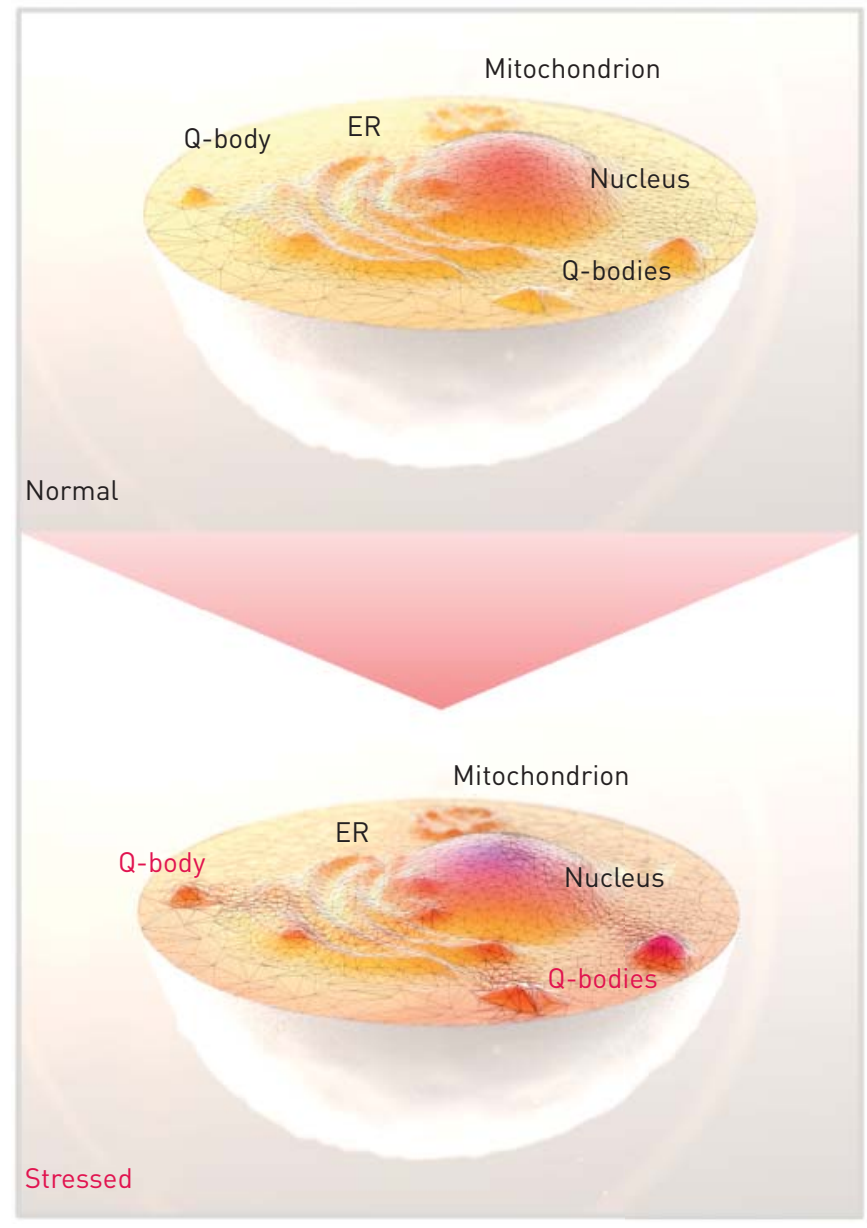

FIGURE 1 The cloud-like proteostasis network in the cell. The proteostasis network is a dynamic and cloud-like multidimensional structure that connects organelle-specific (e.g. nucleus, endoplasmic reticulum (ER) and mitochondria) and cytosolic protein quality compartments (Q-bodies) like a mesh. In the normal cell, this network is well balanced across all compartments, while under conditions of stress, local proteotoxic stress will spread along the mesh-like structure of the proteostasis network and will affect all cellular compartments, as depicted by their coloured and activated state. Extended stress may result in a complete collapse of the proteostasis network of the cell. 


\section{Protein degradation}

Two protein disposal systems, the ubiquitin-proteasome and the autophagy pathways, not only perform "housekeeping" functions of normal protein turnover but also "quality control" tasks that ensure rapid disposal of dysfunctional proteins [17, 18]. For conformational disorders of the lung, the proteasome is the effector disposal system for mutant proteins but its regulation in chronic lung disease has not yet been investigated in detail. Silke Meiners from the Comprehensive Pneumology Center, Munich, introduced the complexity of the proteasome system and how the proteolytic $20 \mathrm{~S}$ proteasome is activated by several regulators in a compartment-specific and dynamic manner [18]. The catalytic activities of the proteasome can be acutely impaired by cigarette smoke exposure [19], leading to a partial collapse of proteostasis that exaggerates protein damage and contributes to lung pathologies. In contrast, proteasome function is enhanced upon myofibroblast differentiation in pulmonary fibrosis, suggesting targeted proteasome inhibition as a novel therapeutic approach for IPF.

A novel perspective on autophagy in chronic lung diseases was provided by emerging data beyond the obvious role of autophagy in protein quality control, on its interaction with cilium function. Patrice Codogno from Paris-Descartes University, Paris, France, presented recent data on how the autophagy pathway regulates ciliary growth by degradation of proteins required for cilium function [20]. These data suggest a close interplay between autophagic protein degradation and cellular nutrient signalling beyond the mammalian target of rapamycin pathway. Suzanne Cloonan, from the laboratory of Augustine Choi (Harvard Medical School, Boston, MA, USA), extended this concept of autophagic removal of ciliary proteins (i.e. "ciliophagy") to ciliated pulmonary cells and defective mucociliary clearance in COPD patients $[21,22]$. Disturbed autophagosome function also involves defective mitochondrial clearance via mitophagy, emphasising the interaction of the different autophagosomal disposal systems in the proteostasis network. Ivan Dikic (Goethe University, Frankfurt, Germany) pointed out that proteasomal and autophagic disposal systems closely interact, as both pathways use ubiquitin for their substrate selection, and critically depend on the solubility of the protein, association with chaperones, its ubiquitination state, or binding to shuttle molecules such as p62 and histone deacetylase 6 [23]. Moreover, the ubiquitin-like LC3 protein serves as a bridging factor for autophagic as well as endosome vesicle formation, indicating a molecular connection between vesicle trafficking and autophagy pathways [24].

\section{Homeostasis of the ER}

A number of conformational disorders of the lung involve the expression of mutant secretory and membrane proteins such as surfactant protein (SP)-C, SP-A, $\alpha_{1}$-AT and CFTR triggering the unfolded protein response (UPR) of the ER [25]. The three UPR sensors, IRE1 $\alpha$, activating transcription factor 6 and PERK, cooperate to restore protein homeostasis in the ER by slowing down translation, activating the folding capacity and increasing degradation of misfolded proteins by proteasomal or autophagic pathways. Stefan Marciniak (Cambridge Institute for Medical Research, Cambridge, UK) showed that some $\alpha_{1}$-AT mutants activate the UPR while others induce ER overload, suggesting that the ER has some degree of freedom in its response to misfolded proteins. Additional stress then tips the balance and makes ER-overloaded cells more sensitive to ER stress responses [26]. Adjustment of protein synthesis to match the folding capacity of the ER is a key event to allow adaptive restoration of protein homeostasis and involves fine-tuned PERK-mediated phosphorylation versus GADD34-induced dephosphorylation of the translation initiation factor eIF2 $\alpha$ [27]. Loss of this negative feedback loop contributes to tumour growth and is detrimental in conditions of chronic ER stress yet offers novel targets for experimental chemotherapeutics [28].

Defects in the processing cascade of secretory proteins represent another challenge to ER homeostasis. Andreas Günther (Universities of Giessen and Marburg Lung Center, Giessen, Germany) provided novel evidence that impaired processing of proSP-B in alveolar epithelial cell type II (AECII) contributes to maladaptive ER stress in sporadic IPF. Reduced expression of the proteases napsin and cathepsin hampered processing of proSP-B, which then accumulated in its unprocessed form and induced ER stress.

While UPR is one way to cope with misfolded or unprocessed secretory proteins, autophagolysosomal disposal of the ER is another option. Michael Beers from the University of Pennsylvania (Philadelphia, PA, USA) showed that autophagy function is impaired in AECII cells of IPF patients in response to missense and splicing mutants of SP-C. In cell culture, abnormalities in autophagosomal protein clearance affected organelle homeostasis not only of the ER but also of mitochondria involving mitophagy, a specialised form of mitochondrial autophagy.

Simon Tavernier, from the research group of Bart Lambrecht (VIB Inflammation Research Center, Ghent, Belgium), added a novel aspect to our understanding of the ER stress response: the IRE1 $\alpha$-X-box binding protein 1 axis regulates homeostasis of $\mathrm{CD}^{+}$dendritic cells in the absence of UPR signalling [29], suggesting a role of UPR effectors in the regulation of cell differentiation beyond mediating a canonical ER stress response. 


\section{Conclusion}

Effective protein quality control is central to lung health. Dysfunctional proteostasis underlies almost all chronic lung diseases and, as such, protein homeostasis represents an essential component of the healthy lung. One key aspect emerging from this ERS workshop is that protein quality control in the lung needs to be understood as a dynamic and multidimensional proteostasis network (fig. 1): the mesh-like proteostasis network connects cloud-like cellular organelles (e.g. the ER, the nucleus and the mitochondria) but also Q-bodies, which serve as local and dynamic quality control sites. Proteotoxic stress dynamically affects multiple cellular compartments and components of the proteostasis network (e.g. parts of the ER, the nucleus and the mitochondrion) and activates protein quality control in Q-bodies coping with misfolded, insoluble and aggregated proteins. Identifying the central hubs that are dysregulated upon proteotoxic stress will be the key to interfering with the dysregulated protein homeostasis that drives pathology of chronic lung diseases. With this new knowledge comes the additional challenge of identifying the idiosyncratic triggers and reactions responsible for specific diseases associated with dysfunctional proteostasis in the lung.

\section{References}

1 Bouchecareilh M, Balch WE. Proteostasis: a new therapeutic paradigm for pulmonary disease. Proc Am Thorac Soc 2011; 8: 189-195.

2 Greene CM, McElvaney NG. Protein misfolding and obstructive lung disease. Proc Am Thorac Soc 2010; 7: 346-355.

3 Tanjore H, Blackwell TS, Lawson WE. Emerging evidence for endoplasmic reticulum stress in the pathogenesis of idiopathic pulmonary fibrosis. Am J Physiol Lung Cell Mol Physiol 2012; 302: L721-L729.

4 Meiners S, Eickelberg O. What shall we do with the damaged proteins in lung disease? Ask the proteasome! Eur Respir J 2012; 40: 1260-1268.

5 Ryter SW, Chen ZH, Kim HP, et al. Autophagy in chronic obstructive pulmonary disease: homeostatic or pathogenic mechanism? Autophagy 2009; 5: 235-237.

6 Mizumura K, Cloonan SM, Haspel JA, et al. The emerging importance of autophagy in pulmonary diseases. Chest 2012; 142: 1289-1299.

7 Wei J, Rahman S, Ayaub EA, et al. Protein misfolding and endoplasmic reticulum stress in chronic lung disease. Chest 2013; 143: 1098-1105.

8 Kim YE, Hipp MS, Bracher A, et al. Molecular chaperone functions in protein folding and proteostasis. Annu Rev Biochem 2013; 82: 323-355.

9 De Boeck K, Kent L, Davies J, et al. CFTR biomarkers: time for promotion to surrogate end-point. Eur Respir J 2013; 41: 203-216.

10 Lebecque P. Trying to find a cure for cystic fibrosis: CFTR biomarkers as outcomes. Eur Respir J 2013; $42: 1155$.

11 Almaça J, Faria D, Sousa M, et al. High-content siRNA screen reveals global ENaC regulators and potential cystic fibrosis therapy targets. Cell 2013; 154: 1390-1400.

12 Ljujic M, Topic A, Nikolic A, et al. Identification of a rare p.G320R alpha-1-antitrypsin variant in emphysema and lung cancer patients. Genet Mol Biol 2010; 33: 5-8.

13 Hipp MS, Patel CN, Bersuker K, et al. Indirect inhibition of $26 \mathrm{~S}$ proteasome activity in a cellular model of Huntington's disease. J Cell Biol 2012; 196: 573-587.

14 Park SH, Kukushkin Y, Gupta R, et al. PolyQ proteins interfere with nuclear degradation of cytosolic proteins by sequestering the Sislp chaperone. Cell 2013; 154: 134-145.

15 Powers ET, Balch WE. Diversity in the origins of proteostasis networks - a driver for protein function in evolution. Nat Rev Mol Cell Biol 2013; 14: 237-248.

16 Roth DM, Balch WE. Q-bodies monitor the quinary state of the protein fold. Nat Cell Biol 2013; 15: 1137-1139.

17 Boya P, Reggiori F, Codogno P. Emerging regulation and functions of autophagy. Nat Cell Biol 2013; 15: 713-720.

18 Meiners S, Keller IE, Semren N, et al. Regulation of the proteasome: evaluating the lung proteasome as a new therapeutic target. Antioxid Redox Signal 2014 [In press DOI: 10.1089/ars.2013.5798].

19 Van Rijt SH, Keller IE, John G, et al. Acute cigarette smoke exposure impairs proteasome function in the lung. Am J Physiol Lung Cell Mol Physiol 2012; 303: L814-L823.

20 Pampliega O, Orhon I, Patel B, et al. Functional interaction between autophagy and ciliogenesis. Nature 2013; 502: 194-200.

21 Cloonan SM, Lam HC, Ryter SW, et al. "Ciliophagy": The consumption of cilia components by autophagy. Autophagy 2014; 10: 532-534.

22 Lam HC, Cloonan SM, Bhashyam AR, et al. Histone deacetylase 6-mediated selective autophagy regulates COPDassociated cilia dysfunction. J Clin Invest 2013; 123: 5212-5230.

Shaid S, Brandts CH, Serve H, et al. Ubiquitination and selective autophagy. Cell Death Differ 2013; 20: 21-30.

Stolz A, Ernst A, Dikic I. Cargo recognition and trafficking in selective autophagy. Nat Cell Biol 2014; 16: 495-501. Wang S, Kaufman RJ. The impact of the unfolded protein response on human disease. J Cell Biol 2012; 197: 857-867. Ordóñez A, Snapp EL, Tan L, et al. Endoplasmic reticulum polymers impair luminal protein mobility and sensitize to cellular stress in alpha1-antitrypsin deficiency. Hepatology 2013; 57: 2049-2060.

27 Van 't Wout EF, Hiemstra PS, Marciniak SJ. The integrated stress response in lung disease. Am J Respir Cell Mol Biol 2014; 44: 1-19.

28 Hetz C, Chevet E, Harding HP. Targeting the unfolded protein response in disease. Nat Rev Drug Discov 2013; 12: 703-719.

29 Osorio F, Tavernier SJ, Hoffmann E, et al. The unfolded-protein-response sensor IRE-1 $\alpha$ regulates the function of CD8 $\alpha^{+}$dendritic cells. Nat Immunol 2014; 15: 248-257. 